\title{
Non-enzymatic antioxidant accumulations in BR-deficient and BR-insensitive barley mutants under control and drought conditions
}

\author{
Damian Gruszka $^{1^{*}}$, Anna Janeczko ${ }^{2}$, Michal Dziurka ${ }^{2}$, Ewa Pociecha ${ }^{3}$, Jozsef Fodor $^{4}$
}

${ }^{1}$ Department of Genetics, Faculty of Biology and Environment Protection, University of Silesia, Katowice, Poland, ${ }^{2}$ The Franciszek Gorski Institute of Plant Physiology, Polish Academy of Sciences, Krakow, Poland, ${ }^{3}$ Department of Plant Physiology, University of Agriculture in Krakow, Krakow, Poland, ${ }^{4}$ Plant Protection Institute, Centre for Agricultural Research, Hungarian Academy of Sciences, Budapest, Hungary

\section{*Corresponding author:}

Dr. Damian Gruszka

Department of Genetics,

Faculty of Biology and Environment Protection,

University of Silesia,

Jagiellonska 28

40-032 Katowice, Poland

damian.gruszka@us.edu.pl

\begin{abstract}
Drought is one of the most adverse stresses that affect plant growth and yield. Disturbances in metabolic activity resulting from drought cause overproduction of Reactive Oxygen Species (ROS). It is postulated that brassinosteroids (BRs) regulate plant tolerance to the stress conditions, but the underlying mechanisms remain largely unknown. An involvement of endogenous BRs in regulation of the antioxidant homeostasis is not fully clarified either. Therefore, the aim of this study was to elucidate the role of endogenous BRs in regulation of non-enzymatic antioxidants in barley (Hordeum vulgare) under control and drought conditions. The plant material included the 'Bowman' cultivar and a group of semi-dwarf Near-Isogenic Lines (NILs), representing mutants deficient in BR biosynthesis or signaling. In general, accumulations of eleven compounds representing various types of nonenzymatic antioxidants were analyzed under both conditions. The analyses of accumulations of reduced and oxidized forms of ascorbate indicated that the BR mutants contain significantly higher
\end{abstract}

This article has been accepted for publication and undergone full peer review but has not been through the copyediting, typesetting, pagination and proofreading process, which may lead to differences between this version and the Version of Record. Please cite this article as doi: 10.1111/ppl.12674

This article is protected by copyright. All rights reserved. 
contents of dehydroascorbic acid under drought conditions when compared with the 'Bowman' cultivar. The analysis of glutathione accumulation indicated that under the control conditions the BRinsensitive NILs contained significantly lower concentrations of this antioxidant when compared with the rest of genotypes. Therefore, we postulate that BR sensitivity is required for normal accumulation of glutathione. A complete accumulation profile of various tocopherols indicated that functional BR biosynthesis and signaling are required for their normal accumulation under both conditions. Results of this study provided an insight into the role of endogenous BRs in regulation of the non-enzymatic antioxidant homeostasis.

\section{Abbreviations}

ABA, abscisic acid; AsA, ascorbic acid; BRs, brassinosteroids; DHA, dehydroascorbate; GSH, reduced form of glutathione; GSSG, oxidized form of glutathione; JA, jasmonic acid; NILs, nearisogenic lines; ROS, reactive oxygen species; SA, salicylic acid

\section{Introduction}

It is known that drought is one of the most adverse and multidimensional environmental stresses that affect plant growth and yield (Osakabe et al. 2014). Plants exposed to drought and other adverse environmental conditions have to coordinate morphological, physiological and biochemical changes in order to adapt to the stress (Bajguz and Hayat 2009, Rao and Chaitanya 2016). Disturbances in metabolic activity occurring as a result of abiotic stresses, including drought, cause overproduction of Reactive Oxygen Species (ROS) and plants need to maintain the redox homeostasis in order to counter the potentially detrimental effects of the enhanced ROS accumulation and oxidative stress (de Carvalho 2008, Gill and Tuteja 2010, Noctor et al. 2014, Choudhury et al. 2016, Raja et al. 2017). Drought and the ensuing increased accumulation of ROS is a major constraint of crop productivity worldwide (Mittler 2002, Apel and Hirt 2004, Reddy et al. 2004, Gill et al. 2013).

ROS, being partially reduced or activated forms of oxygen, are generated endogenously in plant organelles and compartments with high electron transport rates, such as chloroplasts, mitochondria and peroxisomes (Mittler et al. 2011). Hence, ROS are metabolic by-products, and during the course of evolution plants developed regulatory mechanisms of ROS homeostasis (Considine et al. 2015, Dietz 2015, Foyer and Noctor 2016). Moreover, the molecular pathways, which allow precise regulation of a steady state between ROS production and scavenging act in plants to employ ROS as signaling messengers and regulators of physiological processes (Quan et al. 2008, Bajguz and Hayat 2009, Gill et al. 2013, Xia et al. 2015, Dietz et al. 2016, Gilroy et al. 2016, Mignolet-Spruyt et al. 2016). In fact, ROS are commonly produced as a form of plant response to various stresses, therefore ROS represent a point of convergence of various signaling pathways regulating response to the stress factors. The 
integration of various signals through this convergence allows a fine tuning of plant response to environmental conditions, and leads to acclimation (Sewelam et al. 2016, Raja et al. 2017). Mutants impaired in the regulation of ROS homeostasis are more sensitive to abiotic stresses, and unable to mediate a systemic signaling during the stress condition (Mittler et al. 2004, Suzuki et al. 2011, 2013). The complicated molecular mechanisms controlling the ROS accumulation include various enzymatic antioxidants, and operate in various cellular compartments (Foyer and Noctor 2016, Mittler 2016). Apart from the enzymatic antioxidants, plants employ the glutathione-ascorbate cycle (FoyerHaliwell-Asada cycle) and other non-enzymatic antioxidants, which operate in cytosol, mitochondria, plastids and peroxisomes, and regulate the ROS accumulation (Gill and Tuteja 2010, Soares et al. 2016, Raja et al. 2017). However, the extent to which an accumulation of antioxidants is induced by drought is variable and dependent on plant species or even cultivars, plant development and metabolic state, and intensity of the stress (Reddy et al. 2004).

Ascorbic acid (AsA) is the most abundant, versatile and powerful water-soluble antioxidant, which regulates ROS homeostasis, provides protection to membranes, and participates in regeneration of other antioxidants - tocopherols and carotenoids (Reddy et al. 2004, Gill and Tuteja 2010). AsA is also involved in regulation of cell division and expansion, photosynthesis and phytohormone biosynthesis (Kka et al. 2017). Tripeptide glutathione (GSH) is another important component of antioxidant network, participating in maintenance of the redox homeostasis, and present abundantly in various compartments (Foyer and Noctor 2005). It is suggested that GSH may function as a redox sensor since the redox status of the GSH pool is affected by ROS accumulation more intensively that AsA redox status (Foyer and Noctor 2011). GSH is an important component of antioxidant regeneration system, involved in recycling of AsA (de Carvalho 2008, Szarka et al. 2012), takes part in regulation of several physiological processes, and influences expression of stress-responsive genes (Mullineaux and Rausch 2005). Biosynthesis of GSH was reported to be modulated by various factors including stress hormones (Gill et al. 2013). It was reported that drought stimulates accumulation of AsA and GSH (Reddy et al. 2004). The non-enzymatic antioxidants include also tocopherols and tocotrienols, which are lipid-soluble molecules that are essential for maintenance of plastid membrane integrity and their photosynthetic function. Tocopherols and tocotrienols scavenge lipid peroxy radicals, and through concerted action with other antioxidants prevent the propagation of deleterious lipid peroxidation in membranes (Munne-Bosch and Alegre 2002, Hollander-Czytko et al. 2005). Tocopherols and $\beta$-carotene efficiently quench singlet oxygen $\left({ }^{1} \mathrm{O}_{2}\right)$, which is very reactive and detrimental to photosynthetic machinery (Krieger-Liszkay et al. 2008, Gill and Tuteja 2010). Upon oxidation tocopherols and tocotrienols are regenerated by a coordinated action of AsA and GSH, and may reciprocally contribute to recycling of other antioxidants. It was reported that $\alpha$-tocopherol (a predominant form of tocopherols) may regulate concentration of ROS and plant hormones, which control development and stress response. Several reports indicated that water deficit increases $\alpha$ tocopherol concentration in a number of plant species (Munne-Bosch and Alegre 2002, Wu et al. 
2007). Additionally, tocopherol biosynthesis is regulated by several phytohormones, such as jasmonic acid (JA), salicylic acid (SA) and abscisic acid (ABA). It was also reported that AsA, GSH and tocopherols regulate expression of genes associated with abiotic stresses, thus altering acclimation responses. The functions of the AsA-GSH-tocopherol triad are interconnected in a redox network (Szarka et al. 2012).

Phytohormones also belong to key regulators of plant acclimation response to abiotic stresses. Under stress condition an intricate interconnection occurs between phytohormones and ROS (Choudhury et al. 2016). The stress signaling involves ROS and various phytohormones, and these two components converge at the Mitogen-Activated Protein Kinases (MAPKs) level (de Carvalho 2008, Bajguz and Hayat 2009). The elevated concentration of ROS affects gene transcription, proteome, and cellular metabolism, but also modulates hormonal homeostasis, and therefore mediates hormone-dependent growth and the stress tolerance responses (Xia et al. 2015, Choudhury et al. 2016). It is postulated that BRs regulate plant tolerance to various abiotic and biotic stresses, but the mechanisms underlying the BR-dependent stress tolerance remain largely unknown (Raja et al. 2017). However, a link that coordinates drought and BR signaling pathways has been recently identified (Ye et al. 2017). It was reported that BRs induce transcription of a gene encoding NADPH oxidase, and stimulate activity of the enzyme, which leads to increased apoplastic $\mathrm{H}_{2} \mathrm{O}_{2}$ accumulation and modulation of various developmental and stress processes (Nie et al. 2013, Xia et al. 2015). The BRinduced increase in the NADPH oxidase activity and the transient accumulation of $\mathrm{H}_{2} \mathrm{O}_{2}$ lead to stimulation of $\mathrm{ABA}$ biosynthesis. The increase in $\mathrm{ABA}$ biosynthesis is suggested to result in a prolonged $\mathrm{H}_{2} \mathrm{O}_{2}$ accumulation and induction of plant stress tolerance (Zhou et al. 2014). It is suggested that low BR concentration results in a transient ROS production and stomatal opening. On the contrary, high BR content induces a prolonged ROS accumulation, and consequently ABA biosynthesis and stomatal closure (Xia et al. 2014). Therefore, ROS form a convergence point of BR signaling pathway with other phytohormonal and developmental signalosomes (Xia et al. 2015, Raja et al. 2017). Relation between BRs and antioxidant system during drought and osmotic stress has been studied almost exclusively through exogenous BR applications, and in some cases led to ambiguous results (Bajguz and Hayat 2009, Vardhini and Anjum 2015), therefore the role of endogenous BRs in regulation of the antioxidant homeostasis under control and drought stress conditions remains not fully elucidated, especially in crop species (Harb et al. 2015, Rao and Chaitanya 2016, Kka et al. 2017).

Semi-dwarf mutants of cereal crops are of significant importance for modern agriculture because of improved lodging resistance under unfavorable environmental conditions (Gruszka et al. 2011, Dockter and Hansson 2015). Moreover, recently identified barley mutants deficient in BR biosynthesis or signaling may constitute a potential alternative in future breeding programs. The mutant characterisation and functional analysis of the barley genes involved in BR biosynthesis or perception was performed utilizing the very useful tool - Near Isogenic Lines (NILs), which represent a vast collection of mutants, including the semi-dwarf forms deficient in BR metabolism. 
Measurement of the endogenous BR concentrations in these NILs validated that their phenotype is caused by the perturbations in BR biosynthesis or signaling (Dockter et al. 2014). The NILs have been developed by a series of recurrent crossings of original mutants into the genetic background of the barley 'Bowman' cultivar. Later on, the NILs were analyzed with ca. 3000 DNA markers, which provided information on a map position of each introgression region (Druka et al. 2011). This approach allowed for development of the NILs collection where each of the accessions harbors a genomic introgression of known position, specific for each mutant in the genetic background of the 'Bowman' cultivar, which is common to all NILs. This approach enables various comparative physiological analyses (Salvi et al. 2014). Interestingly, recent reports indicate that reduction of BR responses improves plant tolerance to drought (Feng et al. 2015, Northey et al. 2016, Ye et al. 2017). Our recent results also indicated that under drought conditions the semi-dwarf NILs exhibit delayed wilting when compared with a wild-type cultivar (Gruszka et al. 2016). Given the facts that maintenance of ROS homeostasis is crucial for the regulation of plant response to drought, and that ROS form a convergence point of BR signaling pathway with other phytohormonal and developmental signaling networks, it is needed to elucidate the role of endogenous BRs in the regulation of nonenzymatic antioxidant homeostasis in the semi-dwarf barley BR mutants under control and drought conditions. Barley is the fourth most important cereal crop in the world, and research on drought response in this species is of significant importance, especially in the era of global climate change.

\section{Materials and Methods}

\section{Plant material}

The plant material of this study included the barley (Hordeum vulgare) two-rowed spring-type cultivar 'Bowman' and a group of five semi-dwarf Near-Isogenic Lines (NILs), representing barley mutants with defects in BR biosynthesis or signaling. The following NILs deficient in BR biosynthesis were analyzed: BW084 (brh13.p) carrying a missense mutation in the HvCPD gene, BW091 (brh3.g) in which a nonsense mutation in the HvBRD gene was identified, and BW333 (ert-zd.159) harboring a missense mutation in the HvDIM gene (see Appendix S1 in Supporting Information). Additionally, two NILs characterized by abnormalities in BR perception were studied: BW312 (ert-ii.79) and BW885 (uzu1.a), which carry missense mutations in different domains of the HvBRI1 receptor. A detailed description of the analyzed genotypes was published by Dockter et al. (2014). Uniform genetic background of the genotypes enables comparative physiological analyses (Gruszka et al. 2016).

\section{Growth conditions and plant material sampling}


Method of seed germination, controlled conditions of the plant growth and the two-week drought stress condition were recently described by Gruszka et al. (2016). Technical details of the drought stress conditions (including preparation of soil mixture and calculations of the maximum (100\%) soil water capacity) were published by Janeczko et al. (2016). Leaf samples were collected from optimally watered plants as well as from plants after two weeks of drought exposition. During the leaf-tissue samplings plants of all the genotypes were at the same developmental stage (fifth leaf), but samples were prepared from the third and fourth (fully developed) leaves. Extractions and quantifications of the following non-enzymatic antioxidants were performed: AsA, GSH, tocopherols, tocotrienols and $\beta$-carotene. For the quantification of AsA and GSH 0.3-0.6 $\mathrm{g}$ of fresh weight samples were prepared, where one sample contained fragments of leaves from 3 different plants of a given genotype. For the quantification of tocopherols, tocotrienols and $\beta$-carotene $2 \mathrm{~g}$ of fresh weight of leaf samples were collected, where one sample contained leaves from 4-5 different plants of a given genotype. The analyses were performed in 3 replicates for each of the genotypes and growth conditions.

\section{Extraction and quantification of ascorbic acid}

The ascorbate contents were determined in leaf extracts according to the ascorbate oxidase (EC 1.10.3.3) method as described by Foyer et al. (1983). For each sample the leaf tissue was homogenized on ice in $5 \mathrm{ml}$ of $5 \%(\mathrm{w} / \mathrm{v})$ metaphosphoric acid, and centrifuged at $12000 \times \mathrm{g}$ for $15 \mathrm{~min}$ at $4^{\circ} \mathrm{C}$. Once centrifuged, $125 \mu \mathrm{l}$ of the supernatant was adjusted between $\mathrm{pH} 5.5$ and 6.5 by the addition of 25 $\mu \mathrm{l}$ of $1.5 \mathrm{M}$ triethanolamine and $225 \mu \mathrm{l}$ sodium phosphate buffer ( $0.1 \mathrm{M}, \mathrm{pH} 7.4)$. The assay mixture contained $2 \mathrm{ml}$ of sodium phosphate buffer (0.1 M, pH 5.6), $200 \mu \mathrm{l}$ of sample and 1 unit of ascorbate oxidase. The ascorbate content was measured by a decrease of absorbance at $265 \mathrm{~nm}$ in the Ultrospec 2100 pro spectrophotometer (Biosciences Amersham, Sweden) and compared to a standard curve generated with known concentrations of ascorbate.

Dehydroascorbate (DHA) was reduced to ascorbate by using $2 \mathrm{mM}$ dithiothreitol prior to analysis. After an incubation period of $15 \mathrm{~min}$ at room temperature, total ascorbate was determined as above, and DHA content was calculated. All chemicals for the ascorbate assay were purchased from Sigma-Aldrich (St. Louis, MO, USA).

\section{Extraction and quantification of glutathione}

Similarly to the quantification of AsA, concentrations of the reduced and oxidized forms of glutathione (GSH and GSSG, respectively) were determined in the whole-leaf extracts. The leaf material $(0.5 \mathrm{~g} / \mathrm{sample})$ was homogenized in $5 \mathrm{ml}$ of $5 \%(\mathrm{w} / \mathrm{v})$ sulfosalicylic acid with addition of ascorbic acid $(0.45 \mathrm{~g} / 300 \mathrm{ml})$ and EDTA-Na $(0.1116 \mathrm{~g} / 300 \mathrm{ml})$. The samples were centrifuged (4000

This article is protected by copyright. All rights reserved. 
$\mathrm{rpm}, 20 \mathrm{~min}, 4^{\circ} \mathrm{C}$ ), and the obtained supernatants were used for the glutathione analysis. The reduced and oxidized forms of glutathione were determined by a spectrophotometric enzymatic recycling assay with the use of glutathione reductase (GR, EC 1.6.4.2). At first, the extracts $(1000 \mu \mathrm{l})$ were neutralized with $1.5 \mathrm{ml}$ of $0.1 \mathrm{M}$ sodium phosphate buffer $(\mathrm{pH} \mathrm{7.8)}$. The total content of glutathione was quantified by the enzymatic recycling assay which detects both GSSG and GSH according to the protocols by Griffith (1980) and Smith (1985). Addition of GR and NADPH to each sample resulted in an increase in absorbance at the wavelength of $412 \mathrm{~nm}$. For each sample the assay mix contained

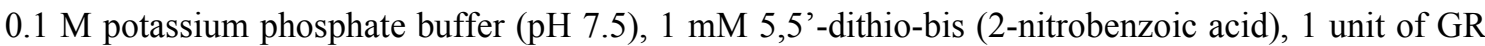
(from Baker's yeast, Type III, Sigma-Aldrich), $0.2 \mathrm{mM} \mathrm{NADPH}$ and $100 \mu 1$ of the neutralized plant extract in the total volume of $3 \mathrm{ml}$. In order to measure the GSSG content, GSH was derivatized by adding $8 \mu 1$ of 2-vinylpyridine to the neutralized samples, upon which the samples were incubated at $25^{\circ} \mathrm{C}$ for 1 hour. GSSG was quantified as described above, by adding $100 \mu 1$ of the sample to the assay mix of the total volume of $3 \mathrm{ml}$. The analyses were performed applying the Ultrospec 2100 pro spectrophotometer (Biosciences Amersham, Sweden). Calibration curves were prepared with the use of GSH stock solutions, and all chemicals were purchased from Sigma-Aldrich (St. Louis, MO, USA).

\section{Extraction and quantification of tocopherols, tocotrienols and $\beta$-carotene}

Extractions of tocopherols, tocotrienols and $\beta$-carotene were performed according to the procedure by Janeczko et al. (2009). Identification and quantification of tocopherols were carried out according to the modified protocol published by Janeczko et al. (2010). The collected plant material ( $2 \mathrm{~g}$ of fresh weight) was lyophilized then pulverized in a mixing mill (MM400, Retsch), and samples of $25 \mathrm{mg}$ of dry weight were prepared for the analysis. The samples were extracted in a mixture of ethanol/acetone/methanol/isopropanol $(8 / 6 / 6 / 1)$ for $15 \mathrm{~min}$ in a water bath at $75^{\circ} \mathrm{C}$. After addition of $250 \mu \mathrm{l}$ of $80 \% \mathrm{KOH}$ the extraction was continued at $75^{\circ} \mathrm{C}$ for $30 \mathrm{~min}$. The samples were diluted with water $(1 / 1)$, centrifuged $2000 \times \mathrm{g}$ at $15^{\circ} \mathrm{C}$ for $5 \mathrm{~min}$, and purified by means of solid supported liquid extraction on Chromabond XTR columns (8.3 g, $45 \mathrm{ml}$, Macherey-Nagel, Düren, Germany). Compounds of interest were eluted with $50 \mathrm{ml}$ of $\mathrm{n}$-hexane. The hexane fraction was evaporated, and the remnants were dissolved in $1.5 \mathrm{ml}$ methanol/methyl chloride $(3 / 1 \mathrm{v} / \mathrm{v})$. Before HPLC analysis the samples were filtered on membranes $(0.22 \mu \mathrm{m}$, SpinX, Corning, USA). Quantification was performed according to the procedure by Biesaga-Koscielniak et al. (2014). The Agilent 1260 binary system with diode array (DAD) and fluorescence (FLD) detectors was used. Separation was achieved on the Ascentis Express C-18 ( $3 \times 150 \mathrm{~mm} ; 2.7 \mu \mathrm{m}$, Supelco, USA) analytical column at $0.8 \mathrm{ml} / \mathrm{min}, 60^{\circ} \mathrm{C}$ and linear gradient of A) acetonitrile/water (6/4 v/v) and B) acetonitrile/isopropanol $(9 / 1 \mathrm{v} / \mathrm{v})$, from 40 to $100 \%$ of $\mathrm{B}$ in $15 \mathrm{~min}$. Tocopherols and tocotrienols were detected by FLD at the excitation wavelength of $295 \mathrm{~nm}$ and the emission wavelength of $330 \mathrm{~nm}$, wears $\beta$-carotene by DAD at $450 \mathrm{~nm}$. Identity and quantity of the monitored compounds was confirmed by comparison with data obtained 
for pure standards at condition identical like for the samples. Pure $\alpha$-tocotrienol, $\gamma$-tocotrienol, $\delta$ tocotrienol, $\alpha$-tocopherol, $\beta$-tocopherol, $\gamma$-tocopherol, $\delta$-tocopherol and $\beta$-carotene were used as standards (Sigma-Aldrich, St. Louis, MO, USA). The analyses of each of three biological replicates were performed on samples representing five individual plants of a given genotype.

\section{Statistical analysis}

Representation of individual plants of a given genotype within each sample and the number of replicates performed for each analysis/measurement were described above. Statistical differences were calculated based on the Duncan test $(P \leq 0.05)$ with use of the Statistica programme. The statistical analyses were performed separately for the optimally-watered plants (control) and the drought-stressed plants. The mean values are presented on the figures together with standard deviations and letters informing about the statistical differences.

\section{Results}

\section{Accumulation profile of ascorbic acid under the control and drought conditions}

Accumulation profiles of the reduced (AsA) and oxidized (DHA) forms of ascorbate were determined in the analyzed genotypes under the control and drought conditions. As far as the AsA accumulation profile is concerned, under the control conditions the highest content of this antioxidant was reported in the 'Bowman' cultivar. In the analyzed NILs the AsA accumulation was reduced to a various degree when compared with the 'Bowman' value. Under the control conditions the AsA accumulation in the NILs ranged between 62\% (in BW885) and 92\% (in BW084) of the reference 'Bowman' value. The drought stress induced an increase in the accumulation of AsA in all analyzed genotypes. However, under the stress conditions the AsA content was significantly $\left(\begin{array}{l}p \\ 0.05)\end{array}\right)$ higher in the 'Bowman' cultivar than in the analyzed NILs (Fig. 1A).

Analysis of the DHA accumulation profile indicated that under the control conditions all analyzed genotypes contained similar concentrations of this form of ascorbate, and the reported differences were found insignificant. Drought induced an increase in the DHA accumulation in all tested genotypes, however an interesting observation was made when the DHA contents were compared between the 'Bowman' cultivar and the mutants. Under the stress conditions the lowest concentration of DHA was reported in the 'Bowman' cultivar, moreover in this genotype the droughtinduced increase with respect to the control value was relatively the lowest $(106 \%$ of the control value). Under the stress conditions the DHA concentrations were significantly ( $p 0.05)$ higher in the mutants when compared with the 'Bowman' value. Moreover, in the analyzed NILs the relative drought-stimulated increases in the DHA accumulation were more pronounced and ranged between ca.

This article is protected by copyright. All rights reserved. 
$227 \%$ and $465 \%$ of the respective control values (Fig. 1B). Interestingly, under the drought conditions the pattern of AsA versus DHA accumulation observed in the 'Bowman' cultivar is the opposite of what was reported in the analyzed BR mutants. Detailed, quantitative results of the AsA and DHA accumulation analyses are given in Supporting Information (Appendix S2, Table S1).

\section{Alterations in a profile of glutathione pool under the control conditions and in reaction to drought}

In this study an accumulation profile of the reduced (GSH) and oxidized (GSSG) forms of glutathione, and a total content of this antioxidant were analyzed under the control and drought conditions. As far as the accumulation profile of GSH is concerned, the contents of this antioxidant were very similar in the 'Bowman' cultivar and the BR biosynthesis-deficient NILs (BW084, BW091 and BW333) under the control conditions. However, the BR-insensitive NILs BW312 and BW885 contained significantly ( $p$ 0.05) lower concentrations of GSH when compared with the 'Bowman' cultivar and the biosynthesis-deficient lines. The BR-insensitive NILs contained ca. $54 \%$ and $62 \%$ of the 'Bowman' GSH content, respectively (Fig. 2A). This indicates that (at least in barley) proper BR sensitivity is required for normal accumulation of GSH under optimal watering conditions. The GSH accumulation pattern was altered in reaction to drought, but to a various extent in the analyzed genotypes. The stress conditions induced a significant increase in the GSH concentration in the 'Bowman' cultivar (ca. $113 \%$ of the respective control value). Drought caused only a slight changes in the GSH concentrations in the BR biosynthesis-deficient NILs. However, the stress conditions induced a significant increase in the GSH concentrations in the BR-insensitive NILs BW312 and BW885 (ca. $149 \%$ and $142 \%$ of the control values, respectively). It should be noted that after drought the GSH contents were similar in the BR mutant NILs, however significantly lower ( $p$ 0.05) when compared with the 'Bowman' cultivar. After drought the mutant NILs contained ca. $69 \%$ - $79 \%$ of the reference 'Bowman' concentration of GSH (Fig. 2A).

As far as the GSSG accumulation profile is concerned, under the control conditions all analyzed genotypes contained similar concentrations of this form, differences were not statistically significant, and no tendency was observed. Under the control conditions the GSSG concentrations were ca. 5 times lower (in BW312, due to the lower concentration of GSH) up to ca. 13 times lower (in BW084) than the GSH content. Drought induced a significant increase in the GSSG accumulation in all analyzed genotypes. After drought the GSSG contents were similar in the analyzed genotypes, and no tendency was observed (Fig. 2B).

The total content of the GSH/GSSG pool was also analyzed. Under the control conditions the total contents of glutathione (GSH and GSSG) were very similar in the 'Bowman' cultivar and the BR biosynthesis-deficient NILs (BW084, BW091 and BW333). However, the BR-insensitive NILs BW312 and BW885 accumulated significantly lower $(p 0.05)$ total contents of the glutathione pool 
(Fig. 2C). Therefore, we conclude that intact BR signaling is required for the glutathione accumulation (predominantly GSH) under the control conditions. Drought induced an increase in the total contents of glutathione in all analyzed genotypes, however various patterns could be observed. A significant drought-stimulated increase in the total content of glutathione was reported in the 'Bowman' cultivar (ca. 125\% of the control value). In the BR biosynthesis-deficient NILs BW084, BW091 and BW333 the drought-induced increases in the total contents of glutathione were relatively less prominent (ca. $102 \%, 112 \%$ and $109 \%$ of the control values, respectively). The drought-induced increases in the total contents of glutathione reported in the BR biosynthesis-deficient NILs were significantly $(p 0.05)$ lower in comparison with the increase reported in the 'Bowman' cultivar. In the BR-insensitive NILs BW312 and BW885 the drought stress resulted in significant increases in the total contents of glutathione (ca. $154 \%$ and $164 \%$ of the control values, respectively). As a consequence of such pronounced increases in the total glutathione contents in the BR-insensitive NILs, after drought all BR mutant NILs accumulated similar contents of the total glutathione pool. However, it should be noted that upon drought the total content of glutathione was significantly higher $(p 0.05)$ in the 'Bowman' cultivar when compared with the rest of genotypes (Fig. 2C). Detailed results of the GSH, GSSG and total glutathione content analyses are given in Supporting Information (Appendix S2, Table S2).

\section{Endogenous brassinosteroids influence accumulation of tocopherols under the control and drought conditions}

In the analyzed genotypes the accumulation profiles of various representatives of tocopherols were determined. In all analyzed genotypes (the 'Bowman' cultivar and BR mutants) $\alpha$-tocopherol proved to be the most abundant form of this group of antioxidants under both control and drought conditions.

However, under the control conditions concentrations of $\alpha$-tocopherol were significantly lower $(p$ 0.05) in the mutant NILs when compared with the 'Bowman' cultivar. The $\alpha$-tocopherol concentrations reported in the analyzed NILs reached $76 \%-86 \%$ of the value reported in the 'Bowman' cultivar. Drought induced a significant increase in the accumulation of $\alpha$-tocopherol in all analyzed genotypes (ca. $118 \%-151 \%$ of the respective control values), however in all NILs the $\alpha$ tocopherol concentrations remained significantly reduced $(p \quad 0.05)$ in comparison with the concentration of this compound in the 'Bowman' cultivar' (Fig. 3A). Under the drought conditions the $\alpha$-tocopherol concentrations reported in the analyzed NILs ranged between ca. $69 \%$ and $79 \%$ of the respective 'Bowman' value.

Measurements of the $\beta$-tocopherol content indicated that this form of tocopherols is accumulated to much lower degree than $\alpha$-tocopherol. Under the control conditions the analyzed genotypes contained similar concentrations of $\beta$-tocopherol. Moreover, the drought stress did not result in any significant change in the $\beta$-tocopherol content in any of the analyzed genotypes, therefore all genotypes contained similar concentrations of this compound (Fig. 3B).

This article is protected by copyright. All rights reserved. 
Analysis of the $\gamma$-tocopherol content indicated that, similarly to $\alpha$-tocopherol, under the control conditions the concentrations of $\gamma$-tocopherol were significantly lower $\left(\begin{array}{ll}p & 0.05\end{array}\right)$ in the mutant NILs when compared with the 'Bowman' cultivar. Under the control conditions the $\gamma$-tocopherol accumulations reported in the analyzed NILs reached ca. $43 \%-68 \%$ of the value reported in the 'Bowman' cultivar. Moreover, the drought stress resulted in a significant increase in the accumulation of $\gamma$-tocopherol in all analyzed genotypes (ca. $157 \%$ - 264\% of the respective control values). However, under the stress conditions in all NILs the $\gamma$-tocopherol concentrations remained significantly lower $\left(\begin{array}{l}p \\ 0.05)\end{array}\right)$ in comparison with the concentration reported in the 'Bowman' cultivar. Under drought the $\gamma$-tocopherol contents in the analyzed NILs reached ca. $38 \%-74 \%$ of the concentration reported in the 'Bowman' cultivar (Fig. 3C).

Quantification of $\delta$-tocopherol in the analyzed genotypes indicated that it is accumulated at the lowest concentrations when compared with other representatives of this group of antioxidants. Under the control conditions all analyzed genotypes contained comparable concentrations of $\delta$-tocopherol (no significant difference and no tendency was observed). Drought did not cause any significant change in the concentration of $\delta$-tocopherol in any of the analyzed genotypes, and under these conditions the $\delta$ tocopherol concentrations were very similar in all analyzed genotypes (Fig. 3D).

This series of analyses allowed for determining a complete profile of accumulation of all representatives of tocopherols in the tested genotypes. Under the control conditions, the mutant NILs (defective in BR biosynthesis or signaling) contained a significantly lower ( $p 0.05)$ total content of tocopherols when compared with the 'Bowman' cultivar. The total content of tocopherols in the NILs reached ca. $76 \%-86 \%$ of the value reported in the 'Bowman' cultivar (Fig. 3E). This is caused mainly by the significantly lower concentrations of $\alpha$-tocopherol and $\gamma$-tocopherol in the mutants with respect to the 'Bowman' cultivar. Drought caused a significant increase in the total content of tocopherols in all analyzed genotypes (ca. 119\% - 154\% of the respective control values), however in the analyzed NILs the total content of tocopherols remained significantly lower $(p 0.05)$ in comparison with the 'Bowman' cultivar (Fig. 3E). The obtained results indicate that endogenous BRs, and proper functioning of BR biosynthesis and signaling, are required for normal accumulation of tocopherols in barley under both control and drought conditions. The analyzed NILs (defective in BR biosynthesis or signaling) contained the significantly lower total contents of tocopherols when compared with the 'Bowman' cultivar under optimal-watering conditions and after drought stress. Although the mutant NILs retained the capacity of increasing the total contents of tocopherols in reaction to drought, the accumulation levels were significantly lower in comparison with the 'Bowman' cultivar. Detailed results of the tocopherol accumulation analyses are given in Supporting Information (Appendix S2, Table S3).

\section{Profiles of accumulation of tocotrienols and $\beta$-carotene}


Apart from the analysis of accumulation of various tocopherols, the profiles of accumulation of two representatives of tocotrienols were also determined. Under the control conditions all analyzed genotypes (the 'Bowman' cultivar and the BR mutants) accumulated very similar concentrations of $\alpha$ tocotrienol (differences were not statistically significant and no tendency was observed). The drought stress did not result in any significant change in the $\alpha$-tocotrienol accumulation in any of the analyzed genotypes, therefore after the stress the concentrations of $\alpha$-tocotrienol remained very similar in all tested genotypes (Fig. 3F).

Another representative of tocotrienols, $\delta$-tocotrienol, was found to be accumulated at much lower concentrations than $\alpha$-tocotrienol. However, similarly to the latter, under the control conditions the accumulation of $\delta$-tocotrienol was very similar in all analyzed genotypes (differences were not statistically significant and no tendency was observed). The accumulation of $\delta$-tocotrienol was not altered by drought, and under the stress conditions all analyzed genotypes accumulated very similar concentrations of $\delta$-tocotrienol (Fig. 3G). Therefore, it may be inferred that, in contrast to tocopherols, the accumulation of tocotrienols is not influenced by the disturbances in BR biosynthesis or signaling, and is not stimulated by drought.

In our study an accumulation profile of another non-enzymatic antioxidant, $\beta$-carotene, was also determined. Relatively, $\beta$-carotene was accumulated at much higher concentrations than any other non-enzymatic antioxidant. Under the control conditions the analyzed genotypes contained similar concentrations of $\beta$-carotene. Interestingly, the drought stress did not cause any increase in the accumulation of $\beta$-carotene in any of the analyzed genotypes. In fact, a decrease in the $\beta$-carotene concentration was reported in every genotype (Fig. 3H). Thus, similarly to tocotrienols, it may be concluded that the accumulation of $\beta$-carotene is not affected by the disturbances in BR biosynthesis or signaling, and is not stimulated by drought. Detailed results of the $\alpha$-tocotrienol, $\delta$-tocotrienol and $\beta$-carotene accumulation analyses are given in Supporting Information (Appendix S2, Table S4).

\section{Discussion}

It was reported in several studies that BRs stimulate activity of antioxidant enzymes (Mazorra et al. 2002, Hasan et al. 2008), however the influence of endogenous BRs on the homeostasis of nonenzymatic antioxidants remains poorly understood. To the best of our knowledge, so far the role of BRs in regulation of antioxidant system during drought stress has been studied almost exclusively through exogenous BR applications, and in some cases led to ambiguous results (Bajguz and Hayat 2009, Vardhini and Anjum 2015). Moreover, it was recently reported that BRs induce apoplastic $\mathrm{H}_{2} \mathrm{O}_{2}$ accumulation (Nie et al. 2013, Xia et al. 2015), therefore analysis of an impact of BRs on the nonenzymatic antioxidant system upon application of exogenous BR may become difficult to interpret because of this secondary $\mathrm{H}_{2} \mathrm{O}_{2}$-mediated effect, and also because the BR-induced accumulation of $\mathrm{H}_{2} \mathrm{O}_{2}$ results in stimulation of $\mathrm{ABA}$ biosynthesis (Zhou et al. 2014). Therefore, the role of endogenous 
BRs in the regulation of non-enzymatic antioxidant homeostasis under the control and drought conditions remains not fully elucidated, especially in crop species (Harb et al. 2015, Kka et al. 2017).

In our study, the semi-dwarf barley NILs defective in BR biosynthesis or signaling were employed to characterize the role of endogenous BRs in the regulation of non-enzymatic antioxidant homeostasis under the control and drought conditions. These mutants may potentially serve as alternatives in future breeding programs (Dockter et al. 2014), and in our recent study exhibited a delayed wilting in reaction to drought when compared with the 'Bowman' cultivar (Gruszka et al. 2016). Taking into account that maintaining the redox homeostasis is pivotal for plant adaptation to drought, the present study was aimed at characterizing the role of endogenous BRs in regulation of this process.

In our study the accumulation profiles of the reduced (AsA) and oxidized (DHA) forms of ascorbate were determined. Under the control conditions the highest content of AsA was reported in the 'Bowman' cultivar, whereas in the analyzed NILs its accumulation was reduced to the various degree when compared with the 'Bowman' value. Drought induced the increase in the accumulation of AsA in all analyzed genotypes, however under the stress conditions the AsA content was significantly higher in the 'Bowman' cultivar than in the analyzed NILs. It may indicate that endogenous BRs regulate AsA homeostasis under both conditions. It should be taken into account that the significantly higher accumulation of AsA reported in the 'Bowman' cultivar may be caused by the more severe effect of the stress condition on plants of this genotype, as it was observed in our recent experiment (Gruszka et al. 2016) and confirmed in this study that the analyzed, semi-dwarf mutant NILs show enhanced tolerance to drought when compared with the 'Bowman' cultivar. However, this observation may be also explained by other results of this study - differences in the DHA accumulation. Analysis of the DHA accumulation profile indicated that under the control conditions all analyzed genotypes contained similar concentrations of this form of ascorbate. Drought induced the increase in the DHA accumulation in all tested genotypes, however under the stress conditions the lowest concentration of DHA was reported in the 'Bowman' cultivar, moreover in this genotype the drought-induced increase (with respect to the control value) was relatively the lowest. In the drought-stressed mutants the DHA concentrations were significantly higher. Moreover, in the mutant NILs the relative drought-stimulated increases in the DHA accumulation were more pronounced. We postulate that this observation may be explained by other results obtained in the present study: under the drought conditions the GSH contents in the BR mutant NILs were significantly lower when compared with the 'Bowman' cultivar. Moreover, during drought the total content of tocopherols also remained significantly lower in the analyzed NILs in comparison with the 'Bowman' cultivar. It should be noted that GSH is an important component of the antioxidant regeneration system, involved in recycling of AsA from DHA. Tocopherols may also contribute to recycling of the other antioxidants (Munne-Bosch and Alegre 2002, de Carvalho 2008, Gill and Tuteja 2010, Szarka et al. 2012). Therefore, we postulate that the differences in the drought-stimulated AsA accumulation reported between the 'Bowman' cultivar and 
the analyzed BR mutants may result from a lower recycling rate of AsA from DHA (the DHA concentrations were significantly higher in the drought-stressed mutants). We suggest that this effect is caused by the reduced accumulations of GSH and tocopherols in the drought-stressed NILs, and therefore it is a secondary effect of the disturbances in BR metabolism. This conclusion is supported by the observation that increase in the accumulation of the antioxidant triad (ascorbate, GSH, tocopherols) occurs in a coordinative manner (Kanwischer et al. 2005).

Analysis of the GSH accumulation profile indicated that under the control conditions the BRinsensitive NILs (BW312 and BW885) contained significantly $(p$ 0.05) lower concentrations of this antioxidant when compared with the 'Bowman' cultivar and the biosynthesis-deficient lines. Therefore, we postulate that (at least in barley) proper BR sensitivity is required for normal accumulation of GSH under optimal watering conditions. It was recently reported that BR signaling and signal transduction pathways of other phytohormones are needed for structural and functional adaptations in reaction to the stress-induced ROS accumulation (Gururani et al. 2015). Our results indicate that the maintenance of GSH homeostasis is dependent on BR signaling also under the control conditions. The GSH accumulation profile was altered in reaction to drought, but to various extent in the analyzed genotypes. The stress conditions induced the significant increase (ca. 113\% of the respective control value) in the GSH concentration in the 'Bowman' cultivar. In our recent research (Gruszka et al. 2016) and in this study the same tendency was reported: in reaction to drought symptoms of leaf wilting were first reported in plants of the 'Bowman' cultivar, whereas the semidwarf NILs exhibited delayed wilting. Similar results were obtained in other recent studies (FerreroSerrano and Assmann 2016, Janeczko et al. 2016, Northey et al. 2016). Moreover, the drought-induced relative decrease in the photosynthesis rate $(\mathrm{Pn})$ value with respect to the control conditions was most prominent in the 'Bowman' cultivar (Gruszka et al. 2016). Therefore, we infer that the significant drought-induced increase in the GSH concentration in the 'Bowman' cultivar results from the more severe effect of the stress conditions on plants of this genotype. This postulate seems to be substantiated by the relatively slight changes in the GSH concentrations, which was observed in the BR biosynthesis-deficient NILs. In this study, drought induced a significant increase in the GSH concentrations in the BR-insensitive NILs (BW312 and BW885). Despite the fact that the BRinsensitive NILs accumulated the significantly lower GSH concentrations under the control conditions, these NILs retained the capacity to react to drought with the significant increase in the GSH contents. This capacity does not seem to be affected by the BR-insensitivity, and upon drought the GSH concentrations were similar in all analyzed NILs. However, it cannot be excluded that the droughtinduced effect is mediated by other phytohormones, especially the stress-related (ABA, SA or JA), as it was recently reported that contents of these hormones are significantly increased in reaction to drought in these genotypes (Gruszka et al. 2016). It should be noted that enzymes involved in glutathione biosynthesis are regulated by drought, ABA, JA and SA (Queval et al. 2009, Gill et al. 2013). Under the control conditions the total contents of glutathione (GSH and GSSG) were very 
similar in the 'Bowman' cultivar and the BR biosynthesis-deficient NILs. However, the BRinsensitive NILs accumulated significantly lower total contents of the glutathione pool. The profile of accumulation of the total glutathione pool resembled the profile of GSH accumulation, because the GSSG contents proved to be similar in all analyzed genotypes. As expected, drought induced the significant increase in the GSSG accumulation in all analyzed genotypes. Thus, the pattern of droughtinduced increase in the total glutathione contents, and the similar contents of the glutathione pools in all drought-stressed NILs indicate that the BR-insensitive NILs, despite the lower contents of GSH and the glutathione pool under the control conditions, retain the capacity for a proportionally higher increase in the accumulation of GSH and the glutathione pool in reaction to drought. This capability may be mediated by other phytohormones, whose contents are induced by drought. The total content of glutathione was significantly higher in the 'Bowman' cultivar when compared with the rest of genotypes, and it may be explained by the more severe effect of the stress on plants of the 'Bowman' cultivar. It is known that contents of glutathione and other non-enzymatic antioxidants are raised in reaction to the stress-induced ROS accumulation (Szarka et al. 2012).

Determining the complete profile of accumulation of all representatives of tocopherols in the tested genotypes led to the conclusion that endogenous BRs, and functional BR biosynthesis and signaling processes, are required for normal accumulation of tocopherols in barley under both control and drought conditions. It results mainly from the significantly lower concentrations of $\alpha$-tocopherol and $\gamma$-tocopherol in the BR mutants with respect to the 'Bowman' cultivar. It is known that biosynthesis of tocopherols is regulated by the stress hormones: ABA, JA and SA (Sandorf and Hollander-Czytko 2002, Munne-Bosch and Penuelas 2003, Munne-Bosch 2005, Szarka et al. 2012), however to the best of our knowledge, up to now an involvement of endogenous BRs in regulation of the tocopherol biosynthesis has not been reported. Our study indicates that functional mechanisms of BR biosynthesis and signaling are crucial for normal accumulation of tocopherols under the control conditions in barley. It is known that the tocopherol biosynthesis from $p$-hydroxyphenyl-pyruvate (HPP) proceeds upon split through two parallel pathways leading to biosynthesis of two final products: $\alpha$-tocopherol and $\beta$-tocopherol (Szarka et al. 2012). Interestingly, the stimulating effect of endogenous BRs on the tocopherol biosynthesis in barley is mediated through modulating effect on one of these pathways, leading through $\gamma$-tocopherol to $\alpha$-tocopherol, which are the most abundant forms of tocopherols in barley (this study) and in Arabidopsis (Shintani and DellaPenna 1998). Under the control conditions, the analyzed mutant NILs accumulated the significantly lower concentrations of $\alpha$ tocopherol and $\gamma$-tocopherol when compared with the 'Bowman' cultivar. However, accumulations of $\beta$-tocopherol and $\delta$-tocopherol, representing the other parallel biosynthesis route, were very similar in all analyzed genotypes. This indicates that, at least in barley, under the control conditions endogenous BRs stimulate the biosynthesis pathway leading to the production of $\gamma$-tocopherol and the most abundant $\alpha$-tocopherol, but not the other route leading to the biosynthesis of $\beta$-tocopherol and $\delta$ tocopherol, which are accumulated at much lower concentrations. Importantly, it was reported that $\alpha$ - 
tocopherol has the highest anti-oxidative activity due to the presence of three methyl groups in its structure (Gill and Tuteja 2010), directly participates in scavenging of the lipid peroxyl radicals, and becomes regenerated by AsA (Szarka et al. 2012). Thus, endogenous BRs regulate the part of the tocopherol biosynthesis pathway leading to the key antioxidant.

It is known that the tocopherol biosynthesis is regulated at its early stage by JA (Falk et al. 2002, Sandorf and Hollander-Czytko 2002). It was reported in our previous study that under the control conditions the analyzed NILs (both BR-deficient and BR-insensitive) accumulated significantly lower concentrations of JA when compared with the 'Bowman' cultivar (Gruszka et al. 2016). However, given the fact that the JA-mediated regulation occurs at the early step of the tocopherol biosynthesis, upstream the divergence of the parallel pathways (Szarka et al. 2012), and since the levels of accumulation of $\beta$-tocopherol and $\delta$-tocopherol are not affected in the analyzed NILs, it seems that the lower concentrations of $\alpha$-tocopherol and $\gamma$-tocopherol are primarily caused by the defects in BR metabolism. Interestingly, it was reported that the tocopherol cyclase (VTE1), catalyzing reactions downstream of the pathway divergence, is a major factor limiting tocopherol biosynthesis in leaves (Kanwischer et al. 2005). This enzyme catalyzes biosynthesis of $\gamma$-tocopherol in one of the parallel routes, which leads to $\alpha$-tocopherol (Szarka et al. 2012). In our study the analyzed mutant NILs accumulated significantly lower concentrations of $\gamma$-tocopherol under the control conditions, and it indicates that, at least in barley, the rate limiting step of tocopherol biosynthesis is dependent on BR homeostasis.

The dependence of tocopherol accumulation on functionality of BR biosynthesis and signaling is illustrated by other results of our study. The drought stress resulted in the significant increase in the total content of tocopherols in all analyzed genotypes. It has been reported that drought and oxidative stresses stimulate accumulation of tocopherols (Wu et al. 2007, Shao et al. 2007). However, under the stress conditions the total contents of tocopherols in the analyzed NILs remained significantly lower when compared with the 'Bowman' cultivar. This effect is caused mainly by the significantly lower concentrations of $\alpha$-tocopherol and $\gamma$-tocopherol in the BR mutants. Our previous research indicated that the abnormalities in BR biosynthesis or signaling in the analyzed mutant NILs result in significantly lower concentrations of the gibberellin $\mathrm{GA}_{7}$ and JA under the control conditions, however after the drought stress the contents of endogenous gibberellins, JA and other phytohormones in the mutant NILs were comparable with the 'Bowman' cultivar or even higher (Gruszka et al. 2016). Taking it into account, the fact that after drought the total contents of tocopherols in the analyzed NILs remained significantly lower than in the 'Bowman' cultivar seems to be a BR deficiency-specific effect. It is known that the tocopherol cyclase (VTE1) is strongly induced by ROS (Kanwischer et al. 2005, Szarka et al. 2012), and our results indicate that endogenous BRs are also crucial for regulation of this part of the tocopherol biosynthesis pathway.

The specific regulatory effect of endogenous BRs on one of the parallel routes of the tocopherol biosynthesis pathway seems to be validated by results of the analysis of tocotrienols accumulation 
profile. Under the control conditions all analyzed genotypes (the 'Bowman' cultivar and BR mutants) accumulated very similar concentrations of $\alpha$-tocotrienol and $\delta$-tocotrienol. Importantly, tocopherols and tocotrienols are synthesized from the common precursor - homogentisate (Norris et al. 1998). Homogentisate is produced in reaction catalyzed by the $p$-hydroxyphenylpyruvate dioxygenase (HPPD), and this reaction is located upstream of the divergence into two parallel routes of the tocopherol biosynthesis pathway (Munne-Bosch and Alegre 2002, Szarka et al. 2012). Since the accumulations of $\alpha$-tocotrienol and $\delta$-tocotrienol are not affected in the analyzed NILs the regulatory effect of endogenous BRs is not located at or upstream the precursor (homogentisate) level, what confirms the conclusion we postulated above.

The results of this study provided an insight into the complicated mechanism of non-enzymatic antioxidant homeostasis in barley, which is an important cereal crop. These results are also a source of further evidence that the drought-stimulated increase in accumulation of the antioxidant triad (ascorbate, GSH, tocopherols) occurs in a coordinated manner. Moreover, the obtained results indicated that functions of the analyzed non-enzymatic antioxidants are interconnected, and that endogenous BRs play an important role in regulation of this process.

\section{Author contributions}

D.G. conceived the project. D.G. and A.J. planned the research. D.G., A.J., E.P., M.D., and J.F. collected and analyzed the data. D.G. interpreted the data and wrote the manuscript. All the authors gave the final approval for submission of the manuscript.

\section{Acknowledgements}

This research was supported by funds from the Polish Ministry of Agriculture and Rural Development (grant no. HOR hn-801/11/14-PO-0114-002).

\section{References}

Apel K, Hirt H (2004) Reactive oxygen species: metabolism, oxidative stress, and signal transduction. Annu Rev Plant Biol 55: 373-399

Bajguz A, Hayat S (2009) Effects of brassinosteroids on the plant responses to environmental stresses. Plant Physiol Biochem 47: 1-8

Biesaga-Koscielniak J, Dziurka M, Ostrowska A, Mirek M, Kościelniak J, Janeczko A (2014) Brassinosteroid improves content of antioxidants in seeds of selected leguminous plants. Aust $\mathrm{J}$ Crop Sci 8: 378-388 
Choudhury FK, Rivero RM, Blumwald E, Mittler M (2016) Reactive oxygen species, abiotic stress and stress combination. Plant J 90: 856-867

Considine MJ, Sandalio LM, Foyer CH (2015) Unravelling how plants benefit from ROS and NO reactions, while resisting oxidative stress. Ann Bot 116: 469-473

de Carvalho MC (2008) Drought stress and reactive oxygen species: production, scavenging and signaling. Plant Signal Behav 3: 156-165

Dietz KJ, Turkan I, Krieger-Liszkay A (2016) Redox- and reactive oxygen species-dependent signaling in and from the photosynthesizing chloroplast. Plant Physiol 171: 1541-1550

Dietz KJ (2015) Efficient high light acclimation involves rapid processes at multiple mechanistic levels. J Exp Bot 66: 2401-2414

Dockter C, Hansson M (2015) Improving barley culm robustness for secured crop yield in a changing climate. J Exp Bot 66: 3499-3509

Dockter C, Gruszka D, Braumann I, Druka A, Druka I, Franckowiak J, Gough SP, Janeczko A, Kurowska M, Lundqvist J, Lundqvist U, Marzec M, Matyszczak I, Müller AH, Oklestkova J, Schulz B, Zakhrabekova S, Hansson M (2014) Induced variations in brassinosteroid genes define barley height and sturdiness, and expand the green revolution genetic toolkit. Plant Physiol 166: 1912-1927

Druka A, Franckowiak J, Lundqvist U, Bonar N, Alexander J, Houston K, Radovic S, Shahinnia F, Vendramin V, Morgante M, Stein N, Waugh R (2011) Genetic dissection of barley morphology and development. Plant Physiol. 155, 617-627

Falk J, Krauss N, Dahnhardt D, Krupinska K (2002) The senescence associated gene of barley encoding 4-hydroxyphenylpyruvate dioxygenase is expressed during oxidative stress. J Plant Physiol 159: 1245-1253

Feng Y, Yin YH, Fei SZ (2015) Down-regulation of BdBRI1, a putative brassinosteroid receptor gene produces a dwarf phenotype with enhanced drought tolerance in Brachypodium distachyon. Plant Sci 234: 163-173

Ferrero-Serrano A, Assmann SM (2016) The $\alpha$-subunit of the rice heterotrimeric G protein, RGA1, regulates drought tolerance during the vegetative phase in the dwarf rice mutant $d 1$. J Exp Bot 67: $3433-3443$

Foyer CH, Noctor G (2005) Redox homeostasis and antioxidant signaling: a metabolic interface between stress perception and physiological responses. Plant Cell 17: 1866-1875

Foyer CH, Noctor G (2011) Ascorbate and glutathione: the heart of the redox hub. Plant Physiol 155: $12-18$

Foyer CH, Noctor G (2016) Stress-triggered redox signaling: what's in pROSpect? Plant Cell Environ 39: 951-964

Foyer CH, Rowell J, Walker D (1983) Measurements of the ascorbate content of spinach leaf protoplasts and chloroplasts during illumination. Planta 157: 239-244

This article is protected by copyright. All rights reserved. 
Gill SS, Tuteja N (2010) Reactive oxygen species and antioxidant machinery in abiotic stress tolerance in crop plants. Plant Physiol Biochem 48: 909-930

Gill SS, Anjum NA, Hasanuzzaman M, Gill R, Trivedi DK, Ahmad I, Pereira E, Tuteja N (2013) Glutathione and glutathione reductase: a boon in disguise for plant abiotic stress defense operations. Plant Physiol Biochem 70: 204-212

Gilroy S, Białasek M, Suzuki N, Górecka M, Devireddy AR, Karpinski S (2016) ROS, calcium, and electric signals: key mediators of rapid systemic signaling in plants. Plant Physiol 171: 16061615

Griffith OW (1980) Determination of glutathione and glutathione disulfide using glutathione reductase and 2-vinylpyridine. Anal Bioch 106: 207-212

Gruszka D, Janeczko A, Dziurka M, Pociecha E, Oklestkova J, Szarejko I (2016) Barley brassinosteroid mutants provide an insight into phytohormonal homeostasis in plant reaction to drought stress. Front Plant Sci 7: 1824

Gruszka D, Szarejko I, Maluszynski M (2011) New allele of HvBRII gene encoding brassinosteroid receptor in barley. J Appl Genet 52: 257-268

Gururani MA, Venkatesh J, Tran LSP (2015) Regulation of photosynthesis during abiotic stressinduced photoinhibition. Mol Plant 8: 1304-1320

Harb A, Awad D, Samarah N (2015) Gene expression and activity of antioxidant enzymes in barley (Hordeum vulgare L.) under controlled severe drought. J Plant Interact 10: 109-116

Hasan SA, Hayat S, Ali B, Ahmad A (2008) 28-Homobrassinolide protects chickpea (Cicer arietinum) from cadmium toxicity by stimulating antioxidants. Environ. Pollut 151: 60-66

Hollander-Czytko H, Grabowski J, Sandorf I, Weckermann K, Weiler EW (2005) Tocopherol content and activities of tyrosine aminotransferase and cystine lyase in Arabidopsis under stress conditions. J Plant Physiol 162: 767-770

Janeczko A, Biesaga-Kościelniak J, Dziurka M (2009) 24-Epibrassinolide modifies seed composition in soybean, oilseed rape and wheat. Seed Sci Technol 37: 625-637

Janeczko A, Biesaga-Kościelniak J, Oklestkova J, Filek M, Dziurka M, Szarek-Łukaszewska G, Kościelniak J (2010) 24-Epibrassinolide in wheat production: physiological activity and uptake. J Agron Crop Sci 196: 311-321

Janeczko A, Gruszka D, Pociecha E, Dziurka M, Filek M, Jurczyk B, Kalaji HM, Kocurek M, Waligorski P (2016) Physiological and biochemical characterisation of watered and droughtstressed barley mutants in the HvDWARF gene encoding C6-oxidase involved in brassinosteroid biosynthesis. Plant Physiol Biochem 99: 126-141

Kanwischer M, Porfirova S, Bergmuller E, Dormann P (2005) Alterations in tocopherol cyclase activity in transgenic and mutant plants of Arabidopsis affect tocopherol content, tocopherol composition, and oxidative stress. J Plant Physiol 137: 713-723

This article is protected by copyright. All rights reserved. 
Kka N, Rookes J, Cahill D (2017) Quantitation of ascorbic acid in Arabidopsis thaliana reveals distinct differences between organs and growth phases. Plant Growth Regul 81: 283-292

Krieger-Liszkay A, Fufezan C, Trebst A (2008) Singlet oxygen production in photosystem II and related protection mechanism. Photosynthesis Res 98: 551-564

Mazorra LM, Núñez M, Hechavarria M, Coll F, Sánchez-Blanco MJ (2002) Influence of brassinosteroids on antioxidant enzymes activity in tomato under different temperatures. Biol Plant 45: 593-596

Mignolet-Spruyt L, Xu E, Idanheimo N, Hoeberichts FA, Muhlenbock P, Brosche M, Van Breusegem F, Kangasjarvi J (2016) Spreading the news: subcellular and organellar reactive oxygen species production and signalling. J Exp Bot 67: 3831-3844

Mittler R, Vanderauwera S, Gollery M, Van Breusegem F (2004) Reactive oxygen gene network of plants. Trends Plant Sci 9: 490-498

Mittler R, Vanderauwera S, Suzuki N, Miller G, Tognetti VB, Vandepoele K, Gollery M, Shulaev V, Van Breusegem F (2011) ROS signaling: the new wave? Trends Plant Sci 16: 300-309

Mittler R (2002) Oxidative stress, antioxidants and stress tolerance. Trends Plant Sci 7: 405-410

Mittler R (2016) ROS are good. Trends Plant Sci 22: 11-19

Mullineaux PM, Rausch T (2005) Glutathione, photosynthesis and the redox regulation of stressresponsive gene expression. Photosynthetic Res 86: 459-474

Munne-Bosch S, Alegre L (2002) The function of tocopherols and tocotrienols in plants. Crit Rev Plant Sci 21: 31-57

Munne-Bosch S, Penuelas J (2003) Photo- and antioxidative protection, and a role for salicylic acid during drought and recovery in field-grown Phillyrea angustifolia plants. Planta 217: 758-766

Munne-Bosch S (2005) The role of $\alpha$-tocopherol in plant stress tolerance. J Plant Physiol 162: 743-748

Nie WF, Wang MM, Xia XJ, Zhou YH, Shi K, Chen Z, Yu JQ (2013) Silencing of tomato RBOH1 and MPK2 abolishes brassinosteroid induced $\mathrm{H}_{2} \mathrm{O}_{2}$ generation and stress tolerance. Plant Cell Environ 36: 789-803

Noctor G, Mhamdi A, Foyer C (2014) Roles of reactive oxygen metabolism in drought: not so cut and dried. Plant Physiol 164: 1636-1648

Norris SR, Shen X, DellaPenna D (1998) Complementation of the Arabidopsis pds1 mutation with the gene encoding $p$-hydroxyphenylpyruvate dioxygenase. J Plant Physiol 117: 1317-1323

Northey JGB, Liang S, Jamshed M, Deb S, Foo E, Reid JB, McCourt P, Samuel MA (2016) Farnesylation mediates brassinosteroid biosynthesis to regulate abscisic acid responses. Nature Plants 2: 16114

Osakabe Y, Osakabe K, Shinozaki K, Tran LSP (2014) Response of plants to water stress. Front Plant Sci $5: 1-8$

Quan LJ, Zhang B, Shi WW, Li HY (2008) Hydrogen peroxide in plants: a versatile molecule of the reactive oxygen species network. J Integrat Plant Biol 50: 2-18

This article is protected by copyright. All rights reserved. 
Queval G, Thominet D, Vanacker H, Miginiac-Maslow M, Gakiere B, Noctor G (2009) $\mathrm{H}_{2} \mathrm{O}_{2}$ activated up-regulation of glutathione in Arabidopsis involves induction of genes encoding enzymes involved in cysteine synthesis in the chloroplast. Mol Plant 2: 344-356

Raja V, Majeed U, Kang H, Andrabi KI, John R (2017) Abiotic stress: Interplay between ROS, hormones and MAPKs. Environ Exp Bot 137: 142-157

Rao DE, Chaitanya KV (2016) Photosynthesis and antioxidative defense mechanisms in deciphering drought stress tolerance of crop plants. Biol Plant 60: 201-218

Reddy AR, Chaitanya KV, Vivekanandan M (2004) Drought-induced responses of photosynthesis and antioxidant metabolism in higher plants. J Plant Physiol 161: 1189-1202

Salvi S, Druka A, Milner S, Gruszka D (2014) Induced genetic variation, TILLING and NGS-based cloning. In: Kumlehn J, Stein N (eds) Biotechnological Approaches to Barley Improvement, Biotechnology in Agriculture and Forestry 69, Springer-Verlag, Berlin Heidelberg, pp 287-310

Sandorf I, Hollander-Czytko H (2002) Jasmonate is involved in the induction of tyrosine aminotransferase and tocopherol biosynthesis in Arabidopsis thaliana. Planta 216: 173-179

Sewelam N, Kazan K, Schenk PM (2016) Global plant stress signaling: reactive oxygen species at the cross-road. Front Plant Sci 7: 187

Shao HB, Chu LY, Wu G, Zhang JH, Lu ZH, Hu YC (2007) Changes of some anti-oxidative physiological indices under soil water deficits among 10 wheat (Triticum aestivum L.) genotypes at tillering stage. Colloid Surf B Biointerfaces 54: 143-149

Shintani D, DellaPenna D (1998) Elevating the vitamin E content of plants through metabolic engineering. Science 282: 2098-2100

Smith IK (1985) Stimulation of glutathione synthesis in photorespiring plants by catalase inhibitors. Plant Physiol 79: 1044-1047

Soares C, de Sousa A, Pinto A, Azenha M, Teixeira J, Azevedo RA, Fidalgo F (2016) Effect of 24epibrassinolide on ROS content, antioxidant system, lipid peroxidation and $\mathrm{Ni}$ uptake in Solanum nigrum L. under Ni stress. Environ Exp Bot 122: 115-125

Suzuki N, Miller G, Morales J, Shulaev V, Torres MA, Mittler R (2011) Respiratory burst oxidases: the engines of ROS signaling. Curr Opin Plant Biol 14: 691-699

Suzuki N, Miller G, Salazar C, Mondal HA, Shulaev E, Cortes DF, Shuman JL, Luo X, Shah J, Schlauch K, Shulaev V, Mittler R (2013) Temporal-spatial interaction between reactive oxygen species and abscisic acid regulates rapid systemic acclimation in plants. Plant Cell 253553 3569

Szarka A, Tomasskovics B, Banhegyi G (2012) The ascorbate-glutathione- $\alpha$-tocopherol triad in abiotic stress response. Int J Mol Sci 13: 4458-4483

Vardhini BV, Anjum NA (2015) Brassinosteroids make plant life easier under abiotic stresses mainly by modulating major components of antioxidant defense system. Front Environ Sci 2: 67

This article is protected by copyright. All rights reserved. 
Wu G, Wei ZK, Wang YX, Chu LY, Shao HB (2007) The mutual responses of higher plants to environment: physiological and microbiological aspects. Colloid Surf B Biointerfaces 59: 113119

Xia XJ, Gao CJ, Song LX, Zhou YH, Shi K, Yu JQ (2014) Role of $\mathrm{H}_{2} \mathrm{O}_{2}$ dynamics in brassinosteroidinduced stomatal closure and opening in Solanum lycopersicum. Plant Cell Environ 379: 20362050

Xia XJ, Zhou YH, Shi K, Zhou J, Foyer CH, Yu JQ (2015) Interplay between reactive oxygen species and hormones in the control of plant development and stress tolerance. J Exp Bot 66: 2839-2856

Ye H, Liu S, Tang B, Chen J, Xie Z, Nolan TM, Jiang H, Guo H, Lin HY, Li L, Wang Y, Tong H, Zhang M, Chu C, Li Z, Aluru M, Aluru S, Schnable PS, Yin Y (2017) RD26 mediates crosstalk between drought and brassinosteroid signalling pathways. Nat. Commun 8: 14573

Zhou J, Wang J, Li X, Xia XJ, Zhou YH, Shi K, Chen Z, Yu JQ (2014) $\mathrm{H}_{2} \mathrm{O}_{2}$ mediates the crosstalk of brassinosteroid and abscisic acid in tomato responses to heat and oxidative stresses. J Exp Bot 65: $4371-4383$

\section{Supporting Information}

Additional Supporting Information may be found in the online version of this article:

Appendix S1. The BR biosynthetic pathway.

Appendix S2. Detailed results of the non-enzymatic antioxidant accumulation analyses.

\section{Figure legends}

Fig. 1. The accumulation of AsA (A) and DHA (B) in the analyzed genotypes under the control and drought conditions. The mean values of three replicates of each measurement are presented for each genotype, with error bars representing standard deviation. The mean values $\pm(\mathrm{SD})$ marked with the same letters (separately for control and drought-stressed plants) are not significantly different, according to the Duncan's test $(P \leq 0.05)$.

Fig. 2. The accumulation of GSH (A), GSSG (B) and the total contents of the glutathione (GSH and GSSG) pool (C) in the analyzed genotypes under the control and drought conditions. The mean values of three replicates of each measurement are presented for each genotype, with error bars representing standard deviation. The mean values $\pm(\mathrm{SD})$ marked with the same letters (separately for control and drought-stressed plants) are not significantly different, according to the Duncan's test $(P \leq 0.05)$.

This article is protected by copyright. All rights reserved. 
Fig. 3. The accumulation of $\alpha$-tocopherol (A), $\beta$-tocopherol (B), $\gamma$-tocopherol (C), $\delta$-tocopherol (D), total tocopherols $(\mathrm{E}), \alpha$-tocotrienol $(\mathrm{F}), \delta$-tocotrienol $(\mathrm{G})$, and $\beta$-carotene $(\mathrm{H})$ in the analyzed genotypes under the control and drought conditions. The mean values of three replicates of each measurement are presented for each genotype, with error bars representing standard deviation. The mean values $\pm(\mathrm{SD})$ marked with the same letters (separately for control and drought-stressed plants) are not significantly different, according to the Duncan's test $(P \leq 0.05)$. 

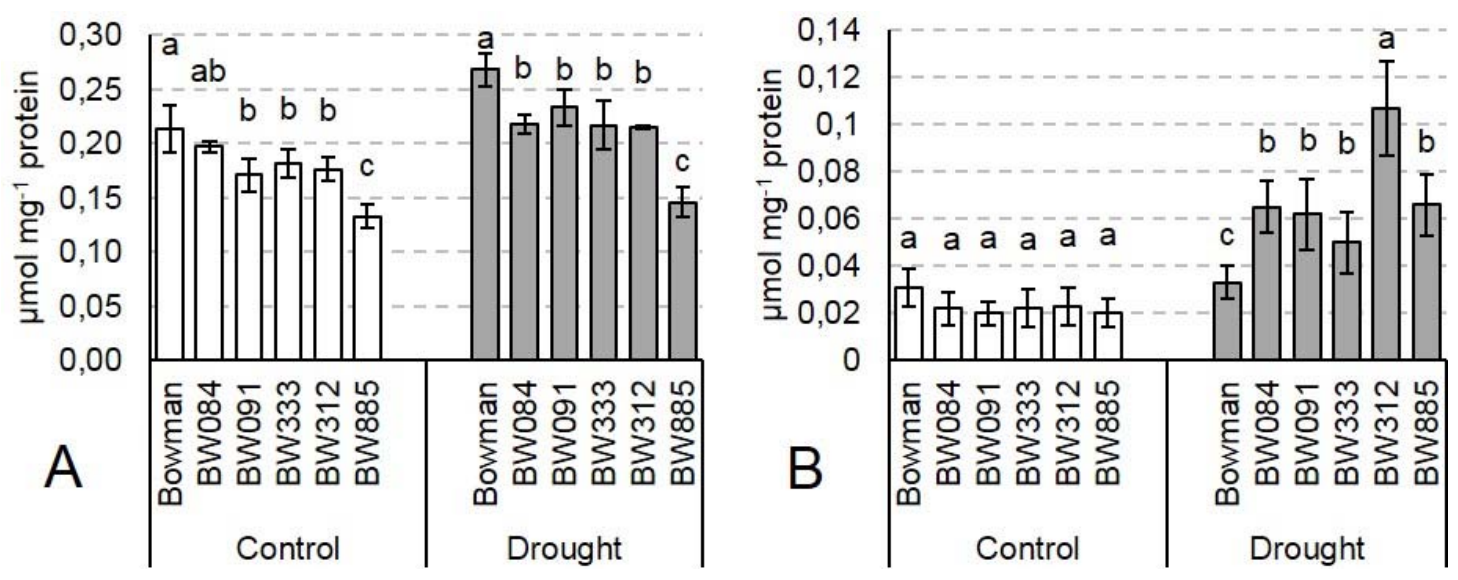


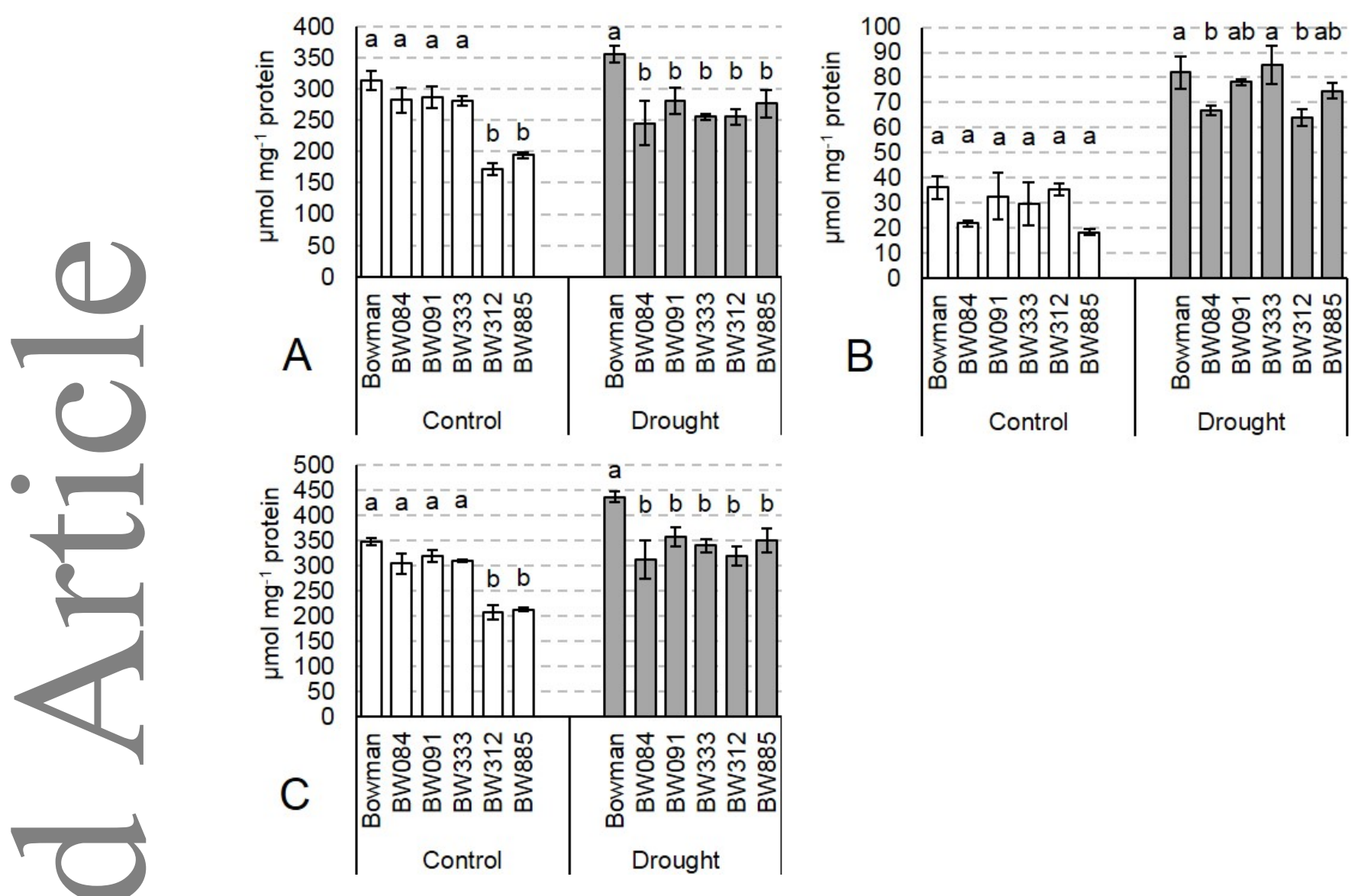



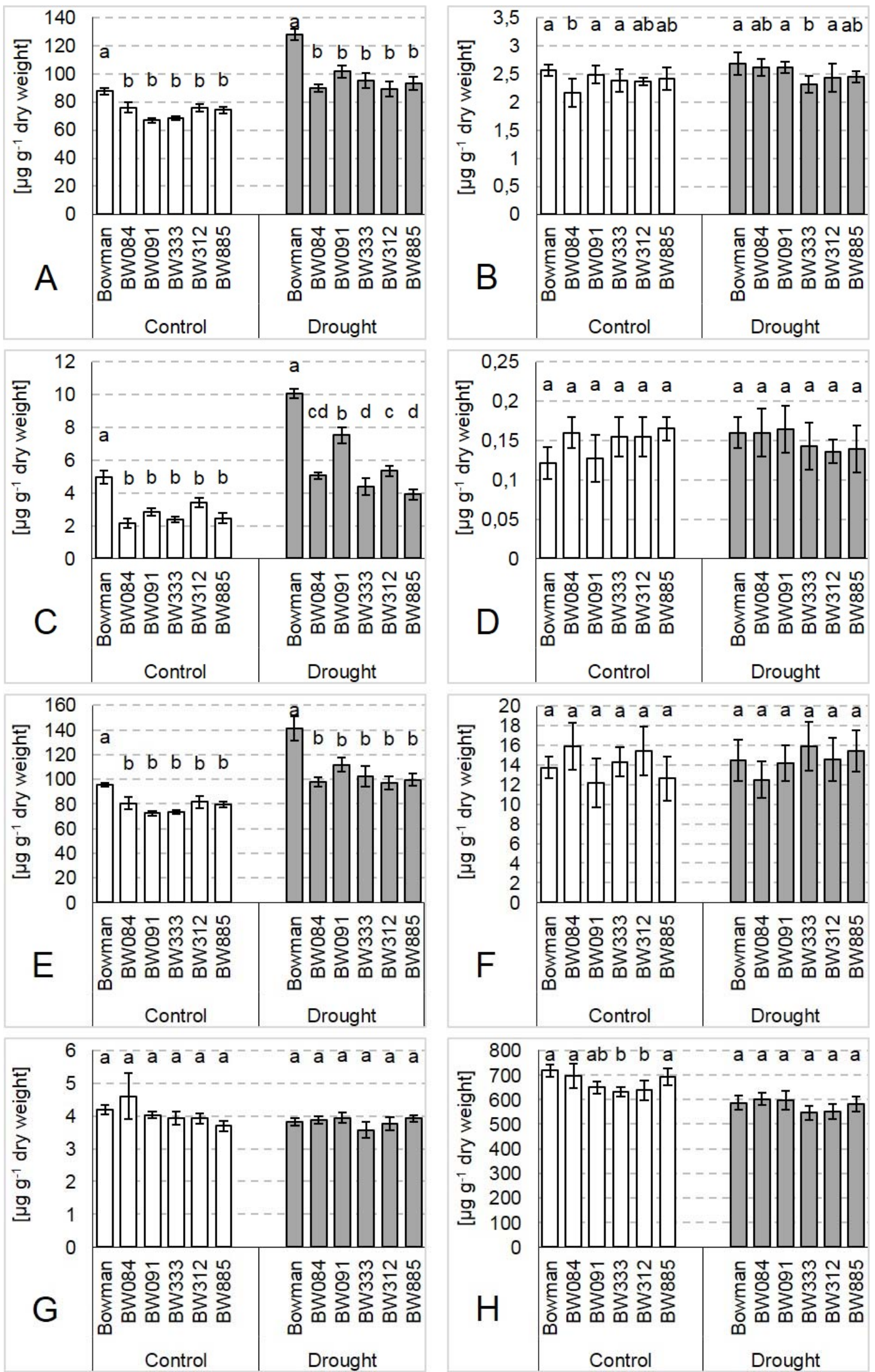